\title{
Foreword
}

\section{Ambulatory Anesthesia: The Innovating Edge of Perioperative} Medicine?

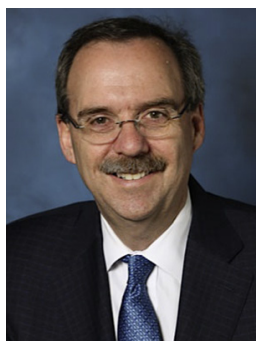

Lee A. Fleisher, MD Consulting Editor

The origin of ambulatory anesthesia can be traced all the way back to 1898 when James Hendson Nicoll performed almost 9000 ambulatory surgical procedures. It took almost 7 decades later before free-standing ambulatory surgery centers were developed. During the past half century, there has been amazing progress in this. These include advances in the preoperative evaluation and preparation of the ambulatory surgery patient and a marked expansion of both the conditions of the patient having surgery as an outpatient and the types of surgery being performed. The advancement of regional and Enhanced Recovery After Surgery techniques has pushed this expansion. Finally, outcomes and metrics for ambulatory surgery and anesthesia are finally being developed. In this issue of the Anesthesiology Clinics, innovations in safely allowing more patients to undergo ambulatory are discussed.

Michael T. Walsh, MD is Assistant Professor of Anesthesiology and a consultant in the Department of Anesthesiology and Perioperative Medicine at the Mayo Clinic. He is the immediate past president of the Society for Ambulatory Anesthesia and the Vice Chair of the Standards Committee of the American Association for 
Accreditation of Ambulatory Surgery Facilities. He is, therefore, well qualified to edit this issue of ambulatory anesthesia.

Lee A. Fleisher, MD Perelman School of Medicine at University of Pennsylvania 3400 Spruce Street, Dulles 680 Philadelphia, PA 19104, USA

E-mail address: Lee.Fleisher@uphs.upenn.edu 